\title{
Dynamic Behavior of Leptospirosis Disease with Saturated Incidence Rate
}

\author{
Muhammad Altaf Khan ${ }^{1}$. Syed Farasat Saddiq ${ }^{2}$. \\ Saeed Islam ${ }^{1}$ - Ilyas Khan ${ }^{3}$. Sharidan Shafie ${ }^{4}$
}

\begin{abstract}
Leptospirosis is a tropical disease and found almost in globe. Human as well as mammals are mostly infected from this disease. This work presents a mathematical study of the leptospirosis disease with saturated incidence rate. Initially, we present the model formulation and their fundamental properties. Then we find the local stability of the disease free and endemic equilibrium. The disease free equilibrium is stable both locally and globally when $R_{0}<1$. Further, we find that endemic equilibrium is stable locally and globally if $R_{0}>1$. The numerical results are shown for analytical results.
\end{abstract}

Keywords Leptospirosis - Saturated incidence - Basic reproduction number - Global stability $\cdot$ Numerical results

Mathematics Subject Classification 92D25 - 49J15 · 93D20

\section{Introduction}

Leptospirosis is a worldwide zoonotic disease which is commonly occurs in tropical and subtropical regions. The magnitude of the problem in the tropics and subtropics may be largely not only because of their climatic and environmental conditions, but also likely relationship

Saeed Islam

saeedislam@awkum.edu.pk

Muhammad Altaf Khan

altafdir@gmail.com

1 Department of Mathematics, Abdul Wali Khan, University Mardan, Mardan, Khyber Pakhtunkhwa, Pakistan

2 Department of Mathematics, Islamia College University Peshawar, Peshawar, Khyber Pakhtunkhwa, Pakistan

3 College of Engineering Majmaah University, Majmaah, Kingdom of Saudi Arabia

4 Department of Mathematical Sciences, Faculty of Science, Universiti Teknologi Malaysia, Skudai, Johor, Malaysia 
with the caused leptospira contaminated environment, such as local agricultural practices and poor housing and disposal, all what will be many sources of infection $[1,2]$. In tropical countries, people are always risky, especially if their contact with potentially infected areas, rodents and pets in a wet and hot climate. In template climate, the cattle, pits and dogs are the carriers for important source of leptospirosis [3]. Valuable information about the risk factor of leptospirosis disease can be found in [4].

To better understand the epidemiology of an infectious disease, mathematical modeling has played an important role [5-8]. These models provide us the quantitative descriptions of the complicated, non linear process of disease transmission and help us to obtain inside into the dynamics of the disease and we are able to make such decision for public health policy. Many mathematical models [9-11] have been proposed to represent the compartmental dynamics of both susceptible, infected and recovered human and vector population.

Various mathematical studies have been proposed on leptospirosis disease. Here, we give an overview. Triampo et al. presented a mathematical model for the transmission of leptospirosis disease in [12]. In their work, they considered a number of leptospirosis disease in Thailand and shown their numerical simulations. Zaman [13] considered the real data presented in [12] to studied the dynamical behavior and role of optimal control theory of this disease. Pongsumpun et al. [14] developed mathematical model to study the behavior of leptospirosis disease. In their work, they represent the rate of change for both the vector (rats) and human population. They divided the human population further into two main groups Juveniles and adults. Moreover, we refer the reader to see [15-17] and the references therein.

In epidemic models a variety of non linear incidence rate have been used [18-20]. Liu et al. [21] showed that the case where hosts can exhibit prolonged immunity to infection is not linear incidence rate of infection could greatly expand the breadth dynamics caused by this disease. The saturated incidence was first time used by Capasso and Serio [20] in cholera epidemic model.

In this work, we present analysis of nonlinear incidence in leptospirosis epidemic model. Here, we study the model presented in [22]. In [22], the authors used the standard optimal control technique Pontryagin's Maximum Principle and used three control variables for possible eradication of the infection in the community. We study the model [22], to obtain its stability analysis on behalf of reproduction number. First, we find the threshold/ basic reproduction number for the model and then show that the Disease free equilibrium is locally as well as globally asymptotically stable for $R_{0}<1$ and unstable for $R_{0}>1$. Further, we find that an endemic equilibrium is locally as well as globally asymptotically stable for $R_{0}>1$. The global stability of an endemic equilibrium is shown by the geometric approach method. We solve, the proposed model numerically and the results are presented in the form of plots for justification purpose. Finally, the conclusion and references are presented.

This paper is organized as follows: section "Mathematical Model" is devoted to the mathematical formulation of the model. We discuss the equilibria and local stability of both the disease-free and endemic equilibrium in section "Equilibria and Local Stability". In section "Global Stability", we show the global stability of both the disease-free and endemic equilibrium. In section "Numerical Simulations" the theoretical results are demonstrated in the form of numerical graphics. Finally, we conclude our work by conclusion in section "Conclusion".

\section{Mathematical Model}

In this section, we present the model formulation of the leptospirosis disease with their interaction with human population. The human population denoted by $N_{h}(t)$, is subdivided 
into three compartments; $S_{h}(t)$-Susceptible individuals, $I_{h}(t)$-infected individuals (those who have been infected) and $R_{h}(t)$-recovered individuals(those who recovered from infection or removed), so

$$
N_{h}(t)=S_{h}(t)+I_{h}(t)+R_{h}(t) .
$$

The population of susceptible is increased by recruitment of the individuals (which is assumed susceptible), at the rate of $b_{h}$. The susceptible population decreased by following the effective contact with the infected individuals and vectors (in the $I_{h}$ and $I_{v}$ ) at a rate $\lambda(t)$, where

$$
\lambda(t)=\left(\frac{\beta_{2} I_{v}}{1+\alpha_{1} I_{v}}+\frac{\beta_{1} I_{h}}{1+\alpha_{2} I_{h}}\right) .
$$

In (2), $\beta_{2}$ and $\beta_{1}$ are the effective contact rate (capable of leading to infection). The population of susceptible is further decreased with the natural death $\mu_{h}$, while increased with the number of individuals, which are susceptible again at a rate of $\lambda_{h}$. So, we can write, the rate of change of the susceptible population given by

$$
\frac{d S_{h}}{d t}=b_{h}-\mu_{h} S_{h}-S_{h} \lambda(t)+\lambda_{h} R_{h} .
$$

The population of infected individuals is generated by the infection of susceptible individuals (at a rate $\lambda(t)$ ). The population of infected individuals is decreased by the natural mortality rate $\mu_{h}$, disease induced death rate $\delta_{h}$ and from the recovery rate of infection $\gamma_{h}$. So, we can write their rate of change for infected population given by

$$
\frac{d I_{h}}{d t}=S_{h} \lambda(t)-\left(\mu_{h}+\delta_{h}+\gamma_{h}\right) I_{h} .
$$

The population of recovered individuals is generated by the rate of recovery $\gamma_{h}$ (from infected class), while decreased by the natural death rate $\mu_{h}$ and $\lambda_{h}$ (those individuals who susceptible again). Thus, their rate of change can be expressed as follows:

$$
\frac{d R_{h}}{d t}=\gamma_{h} I_{h}-\left(\mu_{h}+\lambda_{h}\right) R_{h}
$$

The vector population is denoted by $N_{v}(t)$, is subdivided into two classes, $S_{v}(t)$-susceptible vector and $I_{v}(t)$-infected vector, so

$$
N_{v}(t)=S_{v}(t)+I_{v}(t) .
$$

The population of susceptible vector is generated by the recruitment of the vector at a rate of $b_{v}$. The population of susceptible is decreased by following the effective contact with infected individuals $I_{h}$ (at a rate of $\overline{\lambda(t)}$ ), where $\overline{\lambda(t)}=\beta_{3} I_{h} / 1+\alpha_{2} I_{h}$ and $\beta_{3}$ is the effective contact rate that leads to infection. Also, the population of susceptible vector is decreased by the natural death rate at a rate $\gamma_{v}$. Thus, the rate of change for the susceptible vector is given by

$$
\frac{d S_{v}}{d t}=b_{v}-\gamma_{v} S_{v}-\overline{\lambda(t)} S_{v} .
$$

The population of infected vector is generated by the infection of susceptible vector at rate of $\overline{\lambda(t)}$, while decreased by the natural death $\gamma_{v}$ and disease related death rate $\delta_{v}$. Their rate of change can be written as:

$$
\frac{d I_{v}}{d t}=\overline{\lambda(t)} S_{v}-\gamma_{v} I_{v}-\delta_{v} I_{v} .
$$




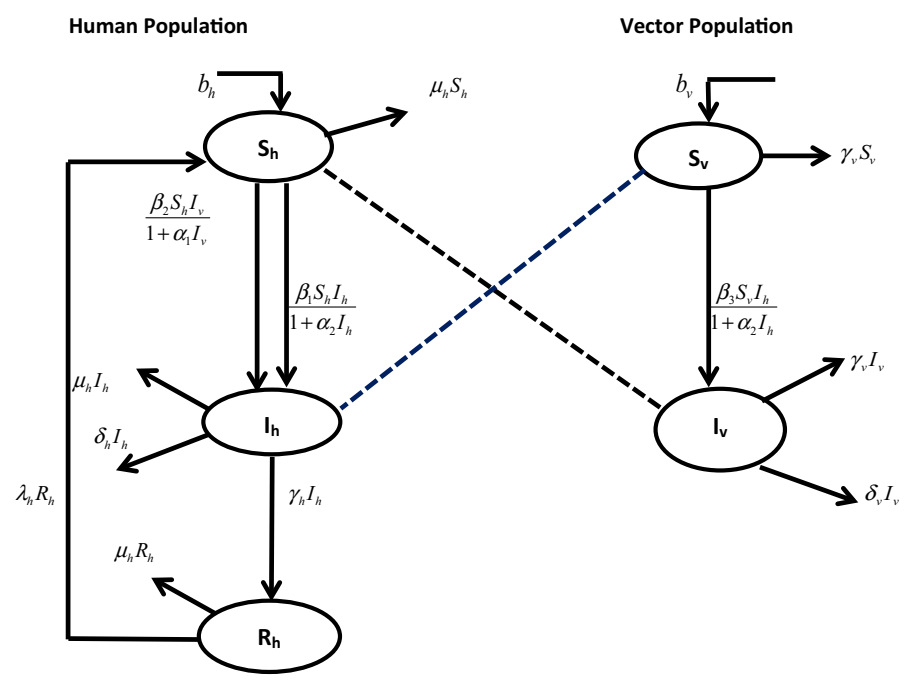

Fig. 1 The flow diagram of human and vector interaction

The complete transfer flow diagram of human and vector population is depicted in Fig. 1. Thus, the system for the transmission dynamics of the leptospirosis disease is given by the following nonlinear system of differential equations:

$$
\begin{aligned}
& \frac{d S_{h}}{d t}=b_{h}-\mu_{h} S_{h}-S_{h} \lambda(t)+\lambda_{h} R_{h}, \quad S_{h}(0)=S_{0} \geq 0, \\
& \frac{d I_{h}}{d t}=S_{h} \lambda(t)-\left(\mu_{h}+\delta_{h}+\gamma_{h}\right) I_{h}, \quad I_{h}(0)=I_{0} \geq 0, \\
& \frac{d R_{h}}{d t}=\gamma_{h} I_{h}-\left(\mu_{h}+\lambda_{h}\right) R_{h}, \quad R_{h}(0)=R_{0} \geq 0, \\
& \frac{d S_{v}}{d t}=b_{v}-\gamma_{v} S_{v}-\overline{\lambda(t)} S_{v}, \quad S_{v}(0)=S_{0} \geq 0, \\
& \frac{d I_{v}}{d t}=\overline{\lambda(t)} S_{v}-\gamma_{v} I_{v}-\delta_{v} I_{v}, \quad I_{v}(0)=I_{0} \geq 0,
\end{aligned}
$$

Subject to nonnegative initial conditions.

One infected human will shed leptospirosis in their urine during and after the illness of the period, and thus may pose a risk of infection to others but only in a certain ways. Chief social interaction is perfectly safe, as airborne bacteria. Saliva is not consider high risky, because the bacteria cannot be tolerate the acidity of the human mouth for a long time, so even we advise against the risk of sharing food, cups or cutlery very small. Items that can dry out between uses, such as towels, also very low risk once they dry, but handle very blood soaked clothing, wet bed or similar may present risks, for detail see the reference [23]. Let $N(h)=S_{h}+I_{h}+R_{h}$, shows the total dynamics of human population at time $t$, is given by

$$
\frac{d N_{h}}{d t}=b_{h}-\mu_{h} N_{h}-\delta_{h} I_{h} \leq b_{h}-\mu_{h} N_{h},
$$


$N(v)=S_{v}+I_{v}$, represent the total population of vector at time $t$, given by

$$
\frac{d N_{v}}{d t}=b_{v}-N_{v} \gamma_{v}-\delta_{v} I_{v} \leq b_{v}-N_{v} .
$$

The feasible region for the system (9) is

$$
\Pi=\left(\left(S_{h}, I_{h}, R_{h}, S_{v}, I_{v}\right) \in \mathbb{R}_{+}^{5}, \quad\left(N_{h} \leq \frac{b_{h}}{\mu_{h}}, N_{v} \leq \frac{b_{v}}{\gamma_{v}}\right)\right) .
$$

It is easy to prove that the set $\Pi$ is positively invariant with respect to system (9).

Lemma 2.1 Let the initial data $S_{h}(0)>0, I_{h}(0)>0, R_{h}(0)>0, S_{v}(0)>0$ and $I_{v}(0)>0$. Then the solution of $S_{h}(t), I_{h}(t), R_{h}(t), S_{v}(t)$ and $I_{v}(t)$ corresponding to model (9), are nonnegative $\forall$ time $t>0$.

Proposition 2.1 Let $\left(S_{h}, I_{h}, R_{h}, S_{v}, I_{v}\right)$, be the solution of the system (9) with non-negative initial conditions and closed set

$\Pi=\left(\left(S_{h}, I_{h}, R_{h}, S_{v}, I_{v}\right) \in R_{+}^{5}, N_{h} \leq \frac{b_{h}}{\mu_{h}}, N_{v} \leq \frac{b_{v}}{\gamma_{v}}\right)$, then $\Pi$ is positively invariant and attracting under the flow described by system (9).

Proof Consider the Lyapunov function

$$
M(t)=\left(N_{h}(t), N_{v}(t)\right)=\left(S_{h}+I_{h}+R_{h}, S_{v}+I_{v}\right) .
$$

The time derivative of Eq. (10) is

$$
\frac{d M}{d t}=\left(b_{h}-\mu_{h} N_{h}-\delta_{h} I_{h}, \quad b_{v}-\gamma_{v} N_{v}-\delta_{v} I_{v}\right) .
$$

Now it is easy to prove that

$$
\begin{array}{lll}
\frac{d N_{h}}{d t} \leq b_{h}-\mu_{h} N_{h} \leq 0 & \text { for } & N_{h} \geq \frac{b_{h}}{\mu_{h}}, \\
\frac{d N_{v}}{d t} \leq b_{v}-\gamma_{v} N_{v} \leq 0 & \text { for } & N_{v} \geq \frac{b_{v}}{\gamma_{v}} .
\end{array}
$$

Thus, it follows that $\frac{d M}{d t} \leq 0$ which implies that $\Pi$ is positively invariant set. Also a standard comparison theorem [24] is used to show that

$$
0 \leq\left(N_{h}, N_{v}\right) \leq\left(\left(N_{h}(0) e^{-\mu_{h} t}+\frac{b_{h}}{\mu_{h}}\left(1-e^{-\mu_{h} t}\right), \quad N_{v}(0) e^{-\gamma_{v} t}+\frac{b_{v}}{\gamma_{v}}\left(1-e^{-\gamma_{v} t}\right)\right) .\right.
$$

Thus as $\mathrm{t} \rightarrow \infty, 0 \leq\left(N_{h}, N_{v}\right) \leq\left(\frac{b_{h}}{\mu_{h}}, \frac{b_{v}}{\gamma_{v}}\right)$ and so $\Pi$ is an attracting and positive invariant set.

\section{Equilibria and Local Stability}

To obtain the endemic equilibria of the system (9), setting the left side of the system (9) equal to zero, we get

$$
\left\{\begin{array}{l}
S_{h}^{*}=\frac{P_{2}\left(1+\alpha_{2} I_{h}^{*}\right)\left(P_{1}\left(\gamma_{v}\left(1+\alpha_{2} I_{h}^{*}\right)+\beta_{3} I_{h}^{*}\right)+\alpha_{1} \beta_{3} b_{v} I_{h}^{*}\right)}{\beta_{2} \beta_{3} b_{v}\left(1+\alpha_{2} I_{h}^{*}\right)+\beta_{1}\left(P_{1}\left(\gamma_{v}\left(1+\alpha_{2} I_{h}^{*}\right)+\beta_{3} I_{h}^{*}\right)+\alpha_{1} \beta_{3} b_{v} I_{h}^{*}\right)}, \\
R_{h}^{*}=\frac{\gamma_{h} I_{h}^{*}}{\left(\mu_{h}+\delta_{h}\right)} \\
S_{v}^{*}=\frac{b_{v}\left(1+\alpha_{2} I_{h}^{*}\right)}{\left(\gamma_{v}\left(1+\alpha_{2} I_{h}^{*}\right)+\beta_{3} I_{h}^{*}\right)} \\
I_{v}^{*}=\frac{\beta_{3} b_{v} I_{h}^{*}}{\left(\gamma_{v}+\delta_{v}\right)\left(\gamma_{v}\left(1+\alpha_{2} I_{h}^{*}\right)+\beta_{3} I_{h}^{*}\right)},
\end{array}\right.
$$


where $P_{1}=\left(\gamma_{v}+\delta_{v}\right), \quad P_{2}=\left(\mu_{h}+\delta_{h}+\gamma_{h}\right), \quad P_{3}=\left(\mu_{h}+\lambda_{h}\right)$.

The disease free equilibrium of the system (9) at $E_{0}$ is

$$
S_{h}^{0}=\frac{b_{h}}{\mu_{h}}, \quad S_{v}^{0}=\frac{b_{v}}{\gamma_{v}} .
$$

The basic reproduction number $R_{0}$ for system (9) is

$$
R_{0}=\frac{b_{h} \beta_{2} \beta_{3} b_{v}+\beta_{1}\left(\gamma_{v}+\delta_{v}\right) b_{h} \gamma_{v}}{\mu_{h}\left(\gamma_{v}+\delta_{v}\right)\left(\mu_{h}+\delta_{h}+\gamma_{h}\right) \gamma_{v}} .
$$

\section{Disease Free Local Stability}

Theorem 3.1 For $R_{0}<1$, the DFE of the model (9) at $E_{0}$ is stable locally asymptotically if $\left(\left(\mu_{h}+\delta_{h}+\gamma_{h}\right)-\beta_{1} b_{h} / \mu_{h}\right)>0$, otherwise unstable.

Proof To show the local stability of the disease free equilibrium, we setting the left side of the system (9), equating to zero, we get the following jacobian matrix $J_{o}$

$$
J_{o}=\left(\begin{array}{ccccc}
-\mu_{h} & -\beta_{1} S_{h}^{o} & \lambda_{h} & 0 & -\beta_{2} S_{h}^{o} \\
0 & \beta_{1} S_{h}^{o}-\left(\mu_{h}+\delta_{h}+\gamma_{h}\right) & 0 & 0 & \beta_{2} S_{h}^{o} \\
0 & \gamma_{h} & -\left(\mu_{h}+\lambda_{h}\right) & 0 & 0 \\
0 & -\beta_{3} S_{v}^{o} & 0 & -\gamma_{v} & 0 \\
0 & \beta_{3} S_{v}^{o} & 0 & 0 & -\left(\gamma_{v}+\delta_{v}\right)
\end{array}\right) .
$$

The characteristics equation to the Jacobian matrix $J_{o}$ is follows as

$$
\left(\mu_{h}+\lambda\right)\left(\gamma_{v}+\lambda\right)\left(\lambda^{3}+a_{1} \lambda^{2}+a_{2} \lambda+a_{3}\right)=0,
$$

where,

$$
\begin{aligned}
a_{1}= & \left(\mu_{h}+\lambda_{h}\right)+\left(\gamma_{v}+\delta_{v}\right)+\left(\mu_{h}+\delta_{h}+\gamma_{h}\right)-\frac{\beta_{1} b_{h}}{\mu_{h}}, \\
a_{2}= & \left(\mu_{h}+\lambda_{h}\right)\left(\left(\mu_{h}+\delta_{h}+\gamma_{h}\right)-\frac{\beta_{1} b_{h}}{\mu_{h}}\right)+\left(\mu_{h}+\lambda_{h}\right)\left(\gamma_{v}+\delta_{v}\right) \\
& +\left(\mu_{h}+\delta_{h}+\gamma_{h}\right)\left(\gamma_{v}+\delta_{v}\right)\left(1-R_{0}\right), \\
& =\left(\mu_{h}+\lambda_{h}\right)\left(\mu_{h}+\delta_{h}+\gamma_{h}\right)\left(\gamma_{v}+\delta_{v}\right)\left(1-R_{0}\right) .
\end{aligned}
$$

Clearly, the two roots of the characteristics equation (14) are $-\mu_{h}$ and $-\gamma_{v}$ have negative real parts. The other roots can be determined from the cubic terms of the characteristics equation (14). Direct calculations by using (15), we get

$$
\begin{aligned}
a_{1} a_{2}-a_{3}= & {\left[\left(\mu_{h}+\lambda_{h}\right)+\left(\gamma_{v}+\delta_{v}\right)+\left(\mu_{h}+\delta_{h}+\gamma_{h}\right)-\frac{\beta_{1} b_{h}}{\mu_{h}}\right] } \\
& \times\left[\left(\mu_{h}+\lambda_{h}\right)\left\{\left(\gamma_{v}+\delta_{v}\right)+\left(\left(\mu_{h}+\delta_{h}+\gamma_{h}\right)-\frac{\beta_{1} b_{h}}{\mu_{h}}\right)\right\}\right] \\
& +\left(\gamma_{v}+\delta_{v}\right)\left(\left(\mu_{h}+\delta_{h}+\gamma_{h}\right)-\frac{\beta_{1} b_{h}}{\mu_{h}}\right)\left(\gamma_{v}+\delta_{v}\right)\left(\mu_{h}+\delta_{h}+\gamma_{h}\right)\left(1-R_{0}\right)>0 .
\end{aligned}
$$


It follows from Routh-Hurwitz crietria, $a_{i}>0$ for $i=1,2,3$ and $a_{1} a_{2}-a_{3}>0$. Thus, all the eigenvalues of the system (9) have negative real parts if $R_{0}<1$ and $\left(\left(\mu_{h}+\delta_{h}+\right.\right.$ $\left.\left.\gamma_{h}\right)-\beta_{1} b_{h} / \mu_{h}\right)>0$. Therefore, the system (9) at the disease free equilibrium $E_{0}$ is locally asymptotically stable, if $R_{0}<1$ and $\left(\left(\mu_{h}+\delta_{h}+\gamma_{h}\right)-\beta_{1} b_{h} / \mu_{h}\right)>0$.

The stability of the Disease free equilibrium of the system (9) for $R_{0}<1$ means that the disease dies out from the community. In this case the endemic equilibrium does not exists but we are interesting to find the properties of the model about the endemic equilibrium point $E_{1}$, when $R_{0}>1$. In the following, we show that the endemic equilibrium point $E_{1}$ of the model (9) is locally asymptotically stable, when $R_{0}>1$.

\section{Local Stability of Endemic Equilibrium}

In this subsection, we discuss the local stability of the model (9) at $E_{1}$, by setting the left hand side of the system (9), equal to zero, we get the Jacobian matrix $J^{*}$ in the following theorem.

Theorem 3.2 For $R_{0}>1$, the endemic equilibrium point $E_{1}$ of the system (9) is locally asymptotically stable if condition (18) and the terms under braces are positive, otherwise unstable.

Proof The Jacobian matrix $J^{*}$ of the system (9) at $E_{1}$ is given by

$$
J^{*}=\left[\begin{array}{ccccc}
-\mu_{h}-\frac{\beta_{2} I_{v}^{*}}{1+\alpha_{1} I_{v}^{*}}-\frac{\beta_{1} I_{h}^{*}}{1+\alpha_{2} I_{h}^{*}} & -\frac{\beta_{1} S_{h}^{*}}{\left(1+\alpha_{2} I_{h}^{*}\right)^{2}} & \lambda_{h} & 0 & -\frac{\beta_{2} S_{h}^{*}}{\left(1+\alpha_{1} I_{v}^{*}\right)^{2}} \\
\frac{\beta_{2} I_{v}^{*}}{1+\alpha_{1} I_{v}^{*}}+\frac{\beta_{1} I_{h}^{*}}{1+\alpha_{2} I_{h}^{*}} & \frac{\beta_{1} S_{h}^{*}}{\left(1+\alpha_{2} I_{h}^{*}\right)^{2}}-\left(\mu_{h}+\delta_{h}+\gamma_{h}\right) & 0 & 0 & \frac{\beta_{2} S_{h}^{*}}{\left(1+\alpha_{1} I_{v}^{*}\right)^{2}} \\
0 & \gamma_{h} & -\left(\mu_{h}+\lambda_{h}\right) & 0 & 0 \\
0 & -\frac{\beta_{3} S_{v}^{*}}{\left(1+\alpha_{2} I_{h}^{*}\right)^{2}} & 0 & -\gamma_{v}-\frac{\beta_{3} I_{h}^{*}}{1+\alpha_{2} I_{h}^{*}} & 0 \\
0 & \frac{\beta_{3} S_{v}^{*}}{\left(1+\alpha_{2} I_{h}^{*}\right)^{2}} & 0 & \frac{\beta_{3} I_{h}^{*}}{1+\alpha_{2} I_{h}^{*}} & -\left(\gamma_{v}+\delta_{v}\right)
\end{array}\right] .
$$

The characteristics equation of (15) is given by

$$
\lambda^{5}+A_{1} \lambda^{4}+A_{2} \lambda^{3}+A_{3} \lambda^{2}+A_{4} \lambda+A_{5}=0
$$

where

$$
\begin{aligned}
A_{1}= & B_{4}+\underbrace{\left(\mu_{h}+\lambda_{h}\right)-\left(B_{1}+B_{3}\right)}+\left(\gamma_{v}+\delta_{v}\right), \\
A_{2}= & B_{4}\left(\gamma_{v}+\delta_{v}\right)+B_{5} B_{6}+(\underbrace{\left(\mu_{h}+\lambda_{h}\right)-\left(B_{1}+B_{3}\right)})\left(B_{4}+\left(\gamma_{v}+\delta_{v}\right)\right) \\
& +\underbrace{\left[\left(B_{2} B_{7}-B_{1} B_{3}\right)-\left(B_{3}+B_{1}\right)\left(\mu_{h}+\lambda_{h}\right)\right]}, \\
A_{3}= & \underbrace{\left(\mu_{h}+\lambda_{h}\right)\left[B_{2} B_{7}-B_{1} B_{3}\right]-B_{2} \gamma_{h} \lambda_{h}}+\underbrace{\left[\left(B_{2} B_{7}-B_{1} B_{3}\right)-\left(B_{3}+B_{1}\right)\left(\mu_{h}+\lambda_{h}\right)\right]} \\
& \times\left[B_{4}+\left(\gamma_{v}+\delta_{v}\right)\right]+[\underbrace{\left(\mu_{h}+\lambda_{h}\right)-\left(B_{1}+B_{3}\right)}] B_{4}\left(\gamma_{v}+\delta_{v}\right)+\left[\left(2 \mu_{h}+\lambda_{h}\right)+\gamma_{v}\right] B_{5} B_{6},
\end{aligned}
$$




$$
\begin{aligned}
A_{4}= & \underbrace{\left\{\left(\mu_{h}+\lambda_{h}\right)\left[B_{2} B_{7}-B_{1} B_{3}\right]-B_{2} \gamma_{h} \lambda_{h}\right\}}\left[B_{4}+\left(\gamma_{v}+\delta_{v}\right)\right] \\
& +B_{4}\left(\gamma_{v}+\delta_{v}\right) \underbrace{\left[\left(B_{2} B_{7}-B_{1} B_{3}\right)-\left(B_{3}+B_{1}\right)\left(\mu_{h}+\lambda_{h}\right)\right]} \\
& +\left[\left(2 \mu_{h}+\lambda_{h}\right) \gamma_{v}+\mu_{h}\left(\mu_{h}+\lambda_{h}\right)\right] B_{5} B_{6}, \\
A_{5}= & B_{4}\left(\gamma_{v}+\delta_{v}\right) \underbrace{\left\{\left(\mu_{h}+\lambda_{h}\right)\left[B_{2} B_{7}-B_{1} B_{3}\right]-B_{2} \gamma_{h} \lambda_{h}\right\}}+B_{5} B_{6} \mu_{h}\left(\mu_{h}+\lambda_{h}\right) \gamma_{v},
\end{aligned}
$$

and

$$
\begin{aligned}
B_{1} & =\mu_{h}+\frac{\beta_{1} I_{h}}{1+\alpha_{2} I_{h}}+\frac{\beta_{2} I_{v}}{1+\alpha_{1} I_{v}}, B_{2}=\frac{\beta_{1} I_{h}}{1+\alpha_{2} I_{h}}+\frac{\beta_{2} I_{v}}{1+\alpha_{1} I_{v}}, \\
B_{3} & =\frac{\beta_{1} S_{h}^{*}}{\left(1+\alpha_{2} I_{h}\right)^{2}}-\left(\mu_{h}+\delta_{h}+\gamma_{h}\right), \\
B_{4} & =\left(\gamma_{v}+\frac{\beta_{3} I_{h}}{1+\alpha_{2} I_{h}}\right), B_{5}=\frac{\beta_{3} S_{v}}{\left(1+\alpha_{2} I_{h}\right)^{2}}, B_{6}=\frac{\beta_{2} S_{h}}{\left(1+\alpha_{1} I_{v}\right)^{2}}, \\
B_{7} & =\frac{\beta_{1} S_{h}}{\left(1+\alpha_{2} I_{h}\right)^{2}}, B_{8}=\frac{\beta_{3} I_{h}}{\left(1+\alpha_{2} I_{h}\right)}
\end{aligned}
$$

The Routh-Hurwitz criteria for (16) is follows as

$$
\begin{aligned}
H_{5}= & \left|\begin{array}{ccccc}
A_{1} & A_{3} & A_{5} & 0 & 0 \\
1 & A_{2} & A_{4} & 0 & 0 \\
0 & A_{1} & A_{3} & A_{5} & 0 \\
0 & 1 & A_{2} & A_{4} & 0 \\
0 & 0 & A_{1} & A_{3} & A_{5}
\end{array}\right|=A_{1} A_{4} A_{5}\left(A_{2} A_{3}-A_{1} A_{4}\right)-A_{1} A_{2}^{2} A_{5}^{2} \\
& +A_{1} A_{4} A_{5}^{2}-A_{4} A_{5}\left(A_{3}^{2}-A_{1} A_{5}\right)+A_{2} A_{3} A_{5}^{2}-A_{5}^{2}>0
\end{aligned}
$$

The eigenvalues of the characteristics equations (16) have negative real parts if $A_{i}>0$ for $i=1,2,3,4,5, H_{5}>0$ and $R_{0}>1$ and the terms under braces in positive. Thus, it follows from Routh-Hurtwiz criteria that the system (9) at the endemic equilibrium point $E_{1}$ is locally asymptotically stable, if $R_{0}>1$ and the terms under braces are positive and the conditions (18) are satisfied.

\section{Global Stability}

In this section, we investigate the global stability of the system (9) at the disease free equilibrium $E_{0}$ and the endemic equilibrium at $E_{2}$. In the following theorem, we first show the disease free global stability for the case $\left(\lambda_{h}=0\right)$.

Theorem 4.1 The system (9) at $E_{0}$ is globally asymptotically stable if $R_{0}<1$, otherwise unstable.

Proof In order to show this result, we construct the following lyapanove function:

$$
\begin{aligned}
L(t)= & \left(\gamma_{v}+\delta_{v}\right)\left(S_{h}-S_{h}^{0}-S_{h}^{0} \log \frac{S_{h}}{S_{h}^{0}}\right)+\left(\gamma_{v}+\delta_{v}\right) I_{h}+\frac{\beta_{2} b_{h}}{\mu_{h}}\left(S_{v}-S_{v}^{0}-S_{v}^{0} \log \frac{S_{v}}{S_{v}^{0}}\right) \\
& +\frac{\beta_{2} b_{h}}{\mu_{h}} I_{v} .
\end{aligned}
$$


Taking the time derivative of (19) along the solutions of system (9), we obtain

$$
\begin{aligned}
L^{\prime}(t)= & \left(\gamma_{v}+\delta_{v}\right)\left(\frac{S_{h}-S_{h}^{0}}{S_{h}}\right)\left[b_{h}-\mu_{h} S_{h}-\left(\frac{\beta_{1} I_{h}}{1+\alpha_{2} I_{h}}+\frac{\beta_{2} I_{v}}{1+\alpha_{1} I_{v}}\right) S_{h}\right] \\
& +\left(\gamma_{v}+\delta_{v}\right)\left[\left(\frac{\beta_{1} I_{h}}{1+\alpha_{2} I_{h}}+\frac{\beta_{2} I_{v}}{1+\alpha_{1} I_{v}}\right) S_{h}-\left(\mu_{h}+\delta_{h}+\gamma_{h}\right) I_{h}\right] \\
& +\frac{\beta_{2} b_{h}}{\mu_{h}}\left(\frac{S_{v}-S_{v}^{0}}{S_{v}}\right)\left[b_{v}-\gamma_{v} S_{v}-\frac{\beta_{3} S_{v} I_{h}}{1+\alpha_{2} I_{h}}\right]+\frac{\beta_{2} b_{h}}{\mu_{h}}\left[\frac{\beta_{3} S_{v} I_{h}}{1+\alpha_{2} I_{h}}\right. \\
& \left.-\left(\gamma_{v}+\delta_{v}\right) I_{v}\right] .
\end{aligned}
$$

Making use of $S_{h}^{0}=\frac{b_{h}}{\mu_{h}}$ and $S_{v}^{0}=\frac{b_{v}}{\gamma_{v}}$ in equation (20), and taking some arrangements, we get

$$
\begin{aligned}
L^{\prime}(t)= & -\mu_{h}\left(\gamma_{v}+\delta_{v}\right) \frac{\left(S_{h}-S_{h}^{0}\right)^{2}}{S_{h}}-\gamma_{v} \frac{\beta_{2} b_{h}}{\mu_{h}} \frac{\left(S_{v}-S_{v}^{0}\right)^{2}}{S_{v}} \\
& -\left(\gamma_{v}+\delta_{v}\right)\left(\mu_{h}+\delta_{h}+\gamma_{h}\right) \alpha_{2} \frac{I_{h}{ }^{2}}{1+\alpha_{2} I_{h}} \\
& -\frac{\left(\gamma_{v}+\delta_{v}\right) \beta_{2} b_{h} \alpha_{1}}{\mu_{h}} \frac{I_{v}{ }^{2}}{1+\alpha_{1} I_{v}}-\frac{I_{h}}{1+\alpha_{2} I_{h}}\left(\gamma+\delta_{v}\right)\left(\mu_{h}+\delta_{h}+\gamma_{h}\right)\left(1-R_{0}\right) .
\end{aligned}
$$

$L^{\prime}(t)$ is negative if $R_{0}<1$ and $L^{\prime}(t)=0$ if $S_{h}=S_{h}^{0}, S_{v}=S_{v}^{0}, I_{h}=I_{v}=0$. Hence, the largest compact invariant set $\left(S_{h}, I_{h}, R_{h}, S_{v}, I_{v}\right) \in \Pi: L^{\prime}(t)=0$ is the singleton set $E_{0}$, where $E_{0}$ is the disease free equilibrium. Thus, by Principle [25], $E_{0}$ is globally asymptotically stable in $\Pi$.

To prove that the endemic equilibrium point $E_{1}$ is globally asymptotically stable, we reduce the system (9), by using $R_{h}=N_{h}-S_{h}-I_{h}$ in first equation of the system (9) and $S_{v}=\frac{b_{v}-\left(\gamma_{v}+\delta_{v}\right) I_{v}}{\gamma_{v}}$ in fifth equation of the system (9), we obtain the following reduced system:

$$
\begin{aligned}
\frac{d S_{h}}{d t} & =b_{h}-\mu_{h} S_{h}-S_{h}\left(\frac{\beta_{2} I_{v}}{1+\alpha_{1} I_{v}}+\frac{\beta_{1} I_{h}}{1+\alpha_{2} I_{h}}\right)+\lambda_{h}\left(N_{h}-S_{h}-I_{h}\right), \\
\frac{d I_{h}}{d t} & =S_{h}\left(\frac{\beta_{2} I_{v}}{1+\alpha_{1} I_{v}}+\frac{\beta_{1} I_{h}}{1+\alpha_{2} I_{h}}\right)-\left(\mu_{h}+\delta_{h}+\gamma_{h}\right) I_{h}, \\
\frac{d I_{v}}{d t} & =\frac{\beta_{3} I_{h}\left(b_{v}-\left(\gamma_{v}+\delta_{v}\right) I_{v}\right)}{\gamma_{v}\left(1+\alpha_{2} I_{h}\right)}-\left(\gamma_{v}+\delta_{v}\right) I_{v},
\end{aligned}
$$

subject to nonnegative initial conditions

$$
S_{h}=S_{h}(0) \geq 0, \quad I_{h}=I_{h}(0) \geq 0, \quad I_{v}=I_{v}(0) \geq 0 .
$$

The endemic equilibrium of the system (22) is denoted by $E_{2}$.

To prove the global stability of the endemic equilibrium, we first give the following lemma.

Lemma If the model $\frac{d x}{d t}=g(x)$, where $g: D \longrightarrow R^{n}$, posses a unique equilibrium $x^{*}$ and also a compact absorbing set exists for $x^{*}$, then $x^{*}$ is stable globally asymptotically given that the function $P(x)$ and a Lozinskii measure $\ell$ exist such that $q=$ limit $_{t \rightarrow \infty}$ sup $\sup _{x} \frac{1}{t} \int_{0}^{t} \ell(B(x(s, x))) d s<0$ [26]. The symbols $P, \ell$ and $B$ will stated in the following result.

Theorem 4.2 If $R_{0}>1$, then the system (22) is globally asymptotically stable at $E_{2}$. 
Proof At $E_{2}$, the Jacobian matrix $J\left(E_{2}\right)$ is given by

$$
J\left(E_{2}\right)=\left(\begin{array}{ccc}
-\mu_{h}-\frac{\beta_{2} I_{v}}{1+\alpha_{1} I_{v}}-\frac{\beta_{1} I_{h}}{1+\alpha_{2} I_{h}}-\lambda_{h} & -\frac{\beta_{1} S_{h}}{\left(1+\alpha_{2} I_{h}\right)^{2}}-\lambda_{h} & -\frac{\beta_{2} S_{h}}{\left(1+\alpha_{1} I_{v}\right)^{2}} \\
\frac{\beta_{2} I_{v}}{1+\alpha_{1} I_{v}}+\frac{\beta_{1} I_{h}}{1+\alpha_{2} I_{h}} & \frac{\beta_{1} S_{h}}{\left(1+\alpha_{2} I_{h}\right)^{2}}-\left(\mu_{h}+\delta_{h}+\gamma_{h}\right) & \frac{\beta_{2} S_{h}}{\left(1+\alpha_{1} I_{v}\right)^{2}} \\
0 & \frac{\beta_{3}\left(b_{v}-\left(\gamma_{v}+\delta_{v}\right) I_{v}\right)}{\gamma_{v}\left(1+\alpha_{2} I_{h}\right)^{2}} & -\frac{\beta_{3} I_{h}\left(\gamma_{v}+\delta_{v}\right)}{\gamma_{v}\left(1+\alpha_{2} I_{h}\right)}-\left(\gamma_{v}+\delta_{v}\right)
\end{array}\right) .
$$

The second additive compound matrix of $J\left(E_{2}\right)$ is denoted by $J^{[2]}$ given by

$$
J^{[2]}=\left(\begin{array}{ccc}
A_{11} & \frac{\beta_{2} S_{h}}{\left(1+\alpha_{1} I_{v}\right)^{2}} & \frac{\beta_{2} S_{h}}{\left(1+\alpha_{1} I_{v}\right)^{2}} \\
\frac{\beta_{3}\left(b_{v}-\left(\gamma_{v}+\delta_{v}\right) I_{v}\right)}{\gamma_{v}\left(1+\alpha_{2} I_{h}\right)^{2}} & A_{22} & -\frac{\beta_{1} S_{h}}{\left(1+\alpha_{2} I_{h}\right)^{2}}-\lambda_{h} \\
0 & \frac{\beta_{2} I_{v}}{1+\alpha_{1} I_{v}}+\frac{\beta_{1} I_{h}}{1+\alpha_{2} I_{h}} & A_{33}
\end{array}\right),
$$

where

$$
\begin{aligned}
& A_{11}=-\mu_{h}-\lambda_{h}-\frac{\beta_{2} I_{v}}{1+\alpha_{1} I_{v}}-\frac{\beta_{1} I_{h}}{1+\alpha_{2} I_{h}}+\frac{\beta_{1} S_{h}}{\left(1+\alpha_{2} I_{h}\right)^{2}}-\left(\mu_{h}+\delta_{h}+\gamma_{h}\right), \\
& A_{22}=-\mu_{h}-\lambda_{h}-\frac{\beta_{2} I_{v}}{1+\alpha_{1} I_{v}}-\frac{\beta_{1} I_{h}}{1+\alpha_{2} I_{h}}-\frac{\beta_{3}\left(\gamma_{v}+\delta_{v}\right) I_{h}}{\gamma_{v}\left(1+\alpha_{2} I_{h}\right)}-\left(\gamma_{v}+\delta_{v}\right), \\
& A_{33}=\frac{\beta_{1} S_{h}}{\left(1+\alpha_{2} I_{h}\right)^{2}}-P_{2}-\frac{\beta_{3}\left(\gamma_{v}+\delta_{v}\right) I_{h}}{\gamma_{v}\left(1+\alpha_{2} I_{h}\right)}-\left(\gamma_{v}+\delta_{v}\right),
\end{aligned}
$$

Consider the function

$$
P=P\left(S_{h}, I_{h}, I_{v}\right)=\operatorname{diag}\left(1, \frac{I_{h}}{I_{v}}, \frac{I_{h}}{I_{v}}\right)
$$

with $P^{-1}$ is

$$
P^{-1}=\operatorname{diag}\left(1, \frac{I_{v}}{I_{h}}, \frac{I_{v}}{I_{h}}\right)
$$

And

$$
P_{f}=\operatorname{diag}\left(0, \frac{I_{v} I_{h}^{\prime}-I_{v}^{\prime} I_{h}}{I_{v}^{2}}, \frac{I_{v} I_{h}^{\prime}-I_{v}^{\prime} I_{h}}{I_{v}^{2}}\right) .
$$

$P_{f} P^{-1}$ is

$$
P_{f} P^{-1}=\operatorname{diag}\left(0, \frac{I_{h}^{\prime}}{I_{h}}-\frac{I_{v}^{\prime}}{I_{v}}, \frac{I_{h}^{\prime}}{I_{h}}-\frac{I_{v}^{\prime}}{I_{v}}\right),
$$

And $P_{f} J^{[2]} P^{-1}$ is

$$
P J^{[2]} P^{-1}=\left(\begin{array}{ccc}
A_{11} & \frac{\beta_{2} S_{h}}{\left(1+\alpha_{1} I_{v}\right)^{2}} \frac{I_{v}}{I_{h}} & \frac{\beta_{2} S_{h}}{\left(1+\alpha_{1} I_{v}\right)^{2}} \frac{I_{v}}{I_{h}} \\
\frac{\beta_{3}\left(b_{v}-\left(\gamma_{v}+\delta_{v}\right) I_{v}\right)}{\gamma_{v}\left(1+\alpha_{2} I_{h}\right)^{2}} \frac{I_{h}}{I_{v}} & A_{22} & -\frac{\beta_{1} S_{h}}{\left(1+\alpha_{2} I_{h}\right)^{2}}-\lambda_{h} \\
0 & \frac{\beta_{2} I_{v}}{1+\alpha_{1} I_{v}}+\frac{\beta_{1} I_{h}}{1+\alpha_{2} I_{h}} & A_{33}
\end{array}\right) .
$$

So, we write

$$
B=P_{f} P^{-1}+P J^{[2]} P^{-1}=\left(\begin{array}{ll}
B_{11} & B_{12} \\
B_{21} & B_{22}
\end{array}\right),
$$


where

$$
\begin{aligned}
B_{11} & =-\mu_{h}-\lambda_{h}-\frac{\beta_{2} I_{v}}{1+\alpha_{1} I_{v}}-\frac{\beta_{1} I_{h}}{1+\alpha_{2} I_{h}}+\frac{\beta_{1} S_{h}}{\left(1+\alpha_{2} I_{h}\right)^{2}}-\left(\mu_{h}+\delta_{h}+\gamma_{h}\right), \\
B_{12} & =\max \left\{\frac{\beta_{2} S_{h}}{\left(1+\alpha_{1} I_{v}\right)^{2}} \frac{I_{v}}{I_{h}}, \frac{\beta_{2} S_{h}}{\left(1+\alpha_{1} I_{v}\right)^{2}} \frac{I_{v}}{I_{h}}\right\}, \\
B_{21} & =\left(\frac{I_{h}}{I_{v}} \frac{\beta_{3}\left(b_{v}-\left(\gamma_{v}+\delta_{v}\right) I_{v}\right)}{\gamma_{v}\left(1+\alpha_{2} I_{h}\right)^{2}}, 0\right)^{T}, \\
B_{22} & =\left(\begin{array}{ll}
T_{11} & T_{12} \\
T_{21} & T_{22}
\end{array}\right),
\end{aligned}
$$

where

$$
\begin{aligned}
& T_{11}=\frac{I_{h}^{\prime}}{I_{h}}-\frac{I_{v}^{\prime}}{I_{v}}-\mu_{h}-\lambda_{h}-\frac{\beta_{2} I_{v}}{1+\alpha_{1} I_{v}}-\frac{\beta_{1} I_{h}}{1+\alpha_{2} I_{h}}-\frac{\beta_{3}\left(\gamma_{v}+\delta_{v}\right) I_{h}}{\gamma_{v}\left(1+\alpha_{2} I_{h}\right)}-\left(\gamma_{v}+\delta_{v}\right), \\
& T_{12}=-\frac{\beta_{1} S_{h}}{\left(1+\alpha_{2} I_{h}\right)^{2}}-\lambda_{h}, \\
& T_{21}=\frac{\beta_{2} I_{v}}{1+\alpha_{1} I_{v}}+\frac{\beta_{1} I_{h}}{1+\alpha_{2} I_{h}}, \\
& T_{22}=\frac{I_{h}^{\prime}}{I_{h}}-\frac{I_{v}^{\prime}}{I_{v}}+\frac{\beta_{1} S_{h}}{\left(1+\alpha_{2} I_{h}\right)^{2}}-\frac{\beta_{3}\left(\gamma_{v}+\delta_{v}\right) I_{h}}{\gamma_{v}\left(1+\alpha_{2} I_{h}\right)}-\left(\gamma_{v}+\delta_{v}\right)-\left(\mu_{h}+\delta_{h}+\gamma_{h}\right) .
\end{aligned}
$$

Suppose the vector $(\hat{u}, \hat{v}, \hat{w})$ in $R^{3}$ and its norm $\|$.$\| will be defined as$

$$
\|(\hat{u}, \hat{v}, \hat{w})\|=\max \{|\hat{u}|,|\hat{v}|+|\hat{w}|\} .
$$

Suppose $\mu B$ represent Lozinski measure with the above defined norm. So as described in [26], we choose

$$
\mu(B) \leq \sup \left(g_{1}, g_{2}\right)
$$

where

$$
g_{1}=\mu\left(B_{11}\right)+\left|B_{12}\right|, \quad g_{2}=\left|B_{21}\right|+\mu\left(B_{22}\right),
$$

$\left|B_{21}\right|$ and $\left|B_{12}\right|$ are the matrix norm associated with vector $\ell$ and $\mu$ represent the Lozinski measure with respect to $\ell$ norm, then

$$
\begin{aligned}
\mu\left(B_{11}\right) & =-\mu_{h}-\lambda_{h}-\frac{\beta_{2} I_{v}}{1+\alpha_{1} I_{v}}-\frac{\beta_{1} I_{h}}{1+\alpha_{2} I_{h}}+\frac{\beta_{1} S_{h}}{\left(1+\alpha_{2} I_{h}\right)^{2}}-\left(\mu_{h}+\delta_{h}+\gamma_{h}\right), \\
\left|B_{12}\right| & =\max \left\{\frac{\beta_{2} S_{h}}{\left(1+\alpha_{1} I_{v}\right)^{2}} \frac{I_{v}}{I_{h}}, \frac{\beta_{2} S_{h}}{\left(1+\alpha_{1} I_{v}\right)^{2}} \frac{I_{v}}{I_{h}}\right\},
\end{aligned}
$$

Therefore,

$$
\begin{aligned}
g_{1} & =\mu\left(B_{11}\right)+\left|B_{12}\right| \\
& =-\mu_{h}-\lambda_{h}-\frac{\beta_{2} I_{v}}{1+\alpha_{1} I_{v}}-\frac{\beta_{1} I_{h}}{1+\alpha_{2} I_{h}}+\frac{\beta_{1} S_{h}}{\left(1+\alpha_{2} I_{h}\right)^{2}}+\frac{\beta_{2} S_{h}}{\left(1+\alpha_{1} I_{v}\right)^{2}} \frac{I_{v}}{I_{h}}-\left(\mu_{h}+\delta_{h}+\gamma_{h}\right), \\
& \leq-\mu_{h}-\lambda_{h}-\frac{\beta_{2} I_{v}}{1+\alpha_{1} I_{v}}-\frac{\beta_{1} I_{h}}{1+\alpha_{2} I_{h}}+\frac{\beta_{1} S_{h}}{\left(1+\alpha_{2} I_{h}\right)}+\frac{\beta_{2} S_{h}}{\left(1+\alpha_{1} I_{v}\right)} \frac{I_{v}}{I_{h}}-\left(\mu_{h}+\delta_{h}+\gamma_{h}\right), \\
& \leq \frac{I_{h}^{\prime}}{I_{h}}-\mu_{h}-\lambda_{h}-\frac{\beta_{2} I_{v}}{1+\alpha_{1} I_{v}}-\frac{\beta_{1} I_{h}}{1+\alpha_{2} I_{h}},
\end{aligned}
$$


using system (22),

$$
\frac{I_{h}^{\prime}}{I_{h}}=\frac{I_{v}}{I_{h}} \frac{\beta_{2} S_{h}}{\left(1+\alpha_{1} I_{v}\right)}+\frac{\beta_{1} S_{h}}{1+\alpha_{2} I_{h}}-\left(\mu_{h}+\delta_{h}+\gamma_{h}\right),
$$

we get

$$
g_{1} \leq \frac{I_{h}^{\prime}}{I_{h}}-\mu_{h}-\lambda_{h}-\frac{\beta_{2} I_{v}}{1+\alpha_{1} I_{v}}-\frac{\beta_{1} I_{h}}{1+\alpha_{2} I_{h}} .
$$

Again

$$
\begin{gathered}
\left|B_{21}\right|=\frac{I_{h}}{I_{v}} \frac{\beta_{3}\left(b_{v}-\left(\gamma_{v}+\delta_{v}\right) I_{v}\right)}{\gamma_{v}\left(1+\alpha_{2} I_{h}\right)^{2}}, \\
\mu\left(B_{22}\right)=\operatorname{Sup}\left\{\frac{I_{h}^{\prime}}{I_{h}}-\frac{I_{v}^{\prime}}{I_{v}}-\mu_{h}-\lambda_{h}-\frac{\beta_{2} I_{v}}{1+\alpha_{1} I_{v}}-\frac{\beta_{1} I_{h}}{1+\alpha_{2} I_{h}}-\frac{\beta_{3}\left(\gamma_{v}+\delta_{v}\right) I_{h}}{\gamma_{v}\left(1+\alpha_{2} I_{h}\right)}\right. \\
-\left(\gamma_{v}+\delta_{v}\right)+\frac{\beta_{2} I_{v}}{1+\alpha_{1} I_{v}}+\frac{\beta_{1} I_{h}}{1+\alpha_{2} I_{h}}, \frac{I_{h}^{\prime}}{I_{h}}-\frac{I_{v}^{\prime}}{I_{v}}+\frac{\beta_{1} S_{h}}{\left(1+\alpha_{2} I_{h}\right)^{2}}-\frac{\beta_{3}\left(\gamma_{v}+\delta_{v}\right) I_{h}}{\gamma_{v}\left(1+\alpha_{2} I_{h}\right)} \\
\left.-\left(\gamma_{v}+\delta_{v}\right)-\left(\mu_{h}+\delta_{h}+\gamma_{h}\right)-\frac{\beta_{1} S_{h}}{\left(1+\alpha_{2} I_{h}\right)^{2}}-\lambda_{h}\right\}, \\
=\frac{I_{h}^{\prime}}{I_{h}}-\frac{I_{v}^{\prime}}{I_{v}}-\mu_{h}-\lambda_{h}-\left(\gamma_{v}+\delta_{v}\right)-\frac{\beta_{3}\left(\gamma_{v}+\delta_{v}\right) I_{h}}{\gamma_{v}\left(1+\alpha_{2} I_{h}\right)}-\left(\mu_{h}+\delta_{h}+\gamma_{h}\right)-\lambda_{h} .
\end{gathered}
$$

So

$$
\begin{aligned}
g_{2}= & \mu\left(B_{22}\right)+\left|B_{21}\right|, \\
= & \frac{I_{h}^{\prime}}{I_{h}}-\frac{I_{v}^{\prime}}{I_{v}}-\mu_{h}-\lambda_{h}-\left(\gamma_{v}+\delta_{v}\right)-\frac{\beta_{3}\left(\gamma_{v}+\delta_{v}\right) I_{h}}{\gamma_{v}\left(1+\alpha_{2} I_{h}\right)} \\
& -\left(\mu_{h}+\delta_{h}+\gamma_{h}\right)-\lambda_{h}+\frac{I_{h}}{I_{v}} \frac{\beta_{3}\left(b_{v}-\left(\gamma_{v}+\delta_{v}\right) I_{v}\right)}{\gamma_{v}\left(1+\alpha_{2} I_{h}\right)^{2}}, \\
\leq & \frac{I_{h}^{\prime}}{I_{h}}-\frac{I_{v}^{\prime}}{I_{v}}-\mu_{h}-\lambda_{h}-\left(\gamma_{v}+\delta_{v}\right)-\frac{\beta_{3}\left(\gamma_{v}+\delta_{v}\right) I_{h}}{\gamma_{v}\left(1+\alpha_{2} I_{h}\right)} \\
& -\left(\mu_{h}+\delta_{h}+\gamma_{h}\right)-\lambda_{h}+\frac{I_{h}}{I_{v}} \frac{\beta_{3}\left(b_{v}-\left(\gamma_{v}+\delta_{v}\right) I_{v}\right)}{\gamma_{v}\left(1+\alpha_{2} I_{h}\right)}, \\
\leq & \frac{I_{h}^{\prime}}{I_{h}}-\mu_{h}-\lambda_{h}-\frac{\beta_{3}\left(\gamma_{v}+\delta_{v}\right) I_{h}}{\gamma_{v}\left(1+\alpha_{2} I_{h}\right)}-\left(\mu_{h}+\delta_{h}+\gamma_{h}\right)-\lambda_{h},
\end{aligned}
$$

we used in above $g_{2}$, the third equation of the system (22),

$$
\frac{I_{v}^{\prime}}{I_{v}}=\frac{I_{h}}{I_{v}} \frac{\beta_{3}\left(b_{v}-\left(\gamma_{v}+\delta_{v}\right) I_{v}\right)}{\gamma_{v}\left(1+\alpha_{2} I_{h}\right)^{2}}-\left(\gamma_{v}+\delta_{v}\right) .
$$

So,

$$
\mu B \leq \sup \left(g_{1}, g_{2}\right) \leq \frac{I_{h}^{\prime}}{I_{h}}-\mu
$$

then,

$$
q=\frac{1}{t} \int_{0}^{t} \mu B d s \leq \frac{1}{t} \int_{0}^{t}\left(\frac{I_{h}^{\prime}}{I_{h}}-\mu\right) d s=\frac{1}{t} \ln \frac{I_{h}(t)}{I_{h}(0)}-\mu .
$$


Table 1 Parameter values used in numerical simulation

\begin{tabular}{|c|c|c|c|}
\hline Notation & Parameter description & Value & References \\
\hline$b_{h}$ & $\begin{array}{l}\text { Recruitment rate for human } \\
\text { population }\end{array}$ & 1.2 & Assumed \\
\hline$\beta_{1}$ & $\begin{array}{l}\text { Transmission rate for human } \\
\text { population }\end{array}$ & 0.04 & Assumed \\
\hline$\beta_{2}$ & $\begin{array}{l}\text { Transmission rate for vector } \\
\text { population }\end{array}$ & 0.04 & Assumed \\
\hline$\beta_{3}$ & Transmission rate between $S_{v}$ and $I_{h}$ & 0.04 & Assumed \\
\hline$\mu_{h}$ & $\begin{array}{l}\text { Natural mortality rate of human } \\
\text { population }\end{array}$ & $4.6 \times 10^{-5}$ & [12] \\
\hline$\lambda_{h}$ & Proportionality constant & $2.85 \times 10^{-3}$ & [12] \\
\hline$\delta_{h}$ & $\begin{array}{l}\text { Disease death rate for human } \\
\text { population }\end{array}$ & $1.0 \times 10^{-3}$ & [28] \\
\hline$\gamma_{v}$ & $\begin{array}{l}\text { Natural mortality rate of vector } \\
\text { population }\end{array}$ & $1.8 \times 10^{-3}$ & [12] \\
\hline$\delta_{v}$ & $\begin{array}{l}\text { Disease death rate for vector } \\
\text { population }\end{array}$ & 0.04 & Assumed \\
\hline$\alpha_{1}$ & $\begin{array}{l}\text { The rate at which the infection force } \\
\text { saturates effect }\end{array}$ & 0.83 & Assumed \\
\hline$\alpha_{2}$ & $\begin{array}{l}\text { The rate at which the infection force } \\
\text { saturates }\end{array}$ & 0.83 & Assumed \\
\hline$b_{v}$ & $\begin{array}{l}\text { Recruitment rate for vector } \\
\text { population }\end{array}$ & 1.3 & Assumed \\
\hline$\gamma_{h}$ & Recovery rate of the infection & $2.7 \times 10^{-3}$ & [28] \\
\hline
\end{tabular}

Implies that $q \leq-\frac{\mu}{2}<0$. Thus the result [27], implies that the positive equilibrium point $E_{2}$ of the system (22) is globally asymptotically stable.

\section{Numerical Simulations}

In this section, we present the numerical solution of the system (9). The parameter values used in the numerical solution are shown in Table 1. Figures 2 and 3 shows the population behavior of human and vector population respectively. Figures 4, 5, 6, 7 and 8 represents Susceptible human, infected human, recovered human, susceptible vector and infected vector with the parameters $\alpha_{1}$ and $\alpha_{2}$. Figure 2 represent the human population, the dashed line represent the behavior of susceptible human, the bold line shows the population behavior of infected individuals, the dot dashed line shows the population behavior of recovered human. Figure 3 shows the population of behavior of vector, the susceptible vector is represented by bold line and the dashed line shows the population of infected vector. The plot shows the susceptible human population with saturation effect for the values of $\alpha_{1}$ and $\alpha_{2}$ in Fig. 4. The graph of susceptible human decreases with the saturated effect, for the values of $\alpha_{1}=0.83$ and $\alpha_{2}=0.83$. The dotted line shows the population of susceptible human without saturation effect and the dashed line shows the saturation effect of human and the saturation effect of vector is represented by a dot dashed line. Due the effect of $\alpha_{1}$ and $\alpha_{2}$ the population of 


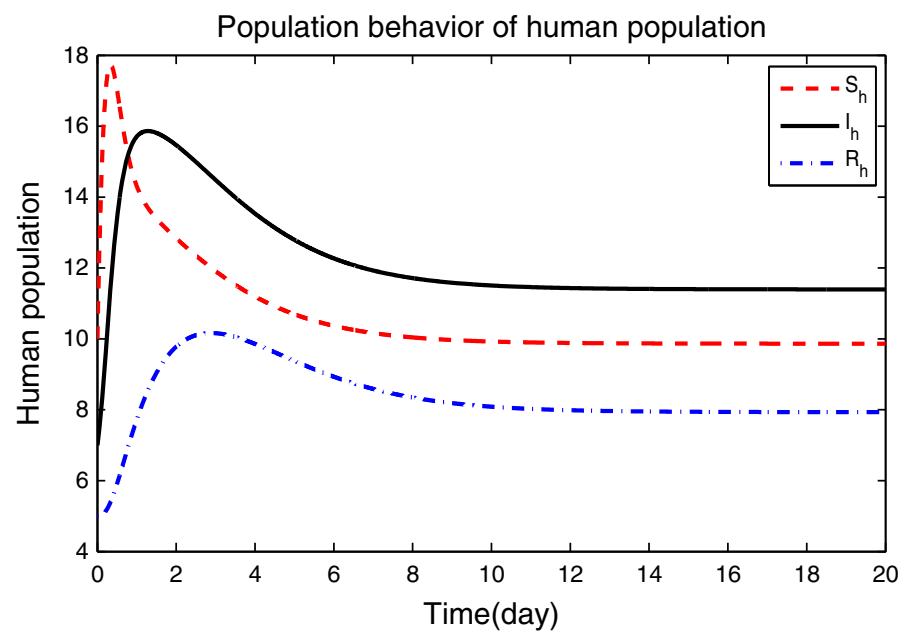

Fig. 2 Population behavior of human

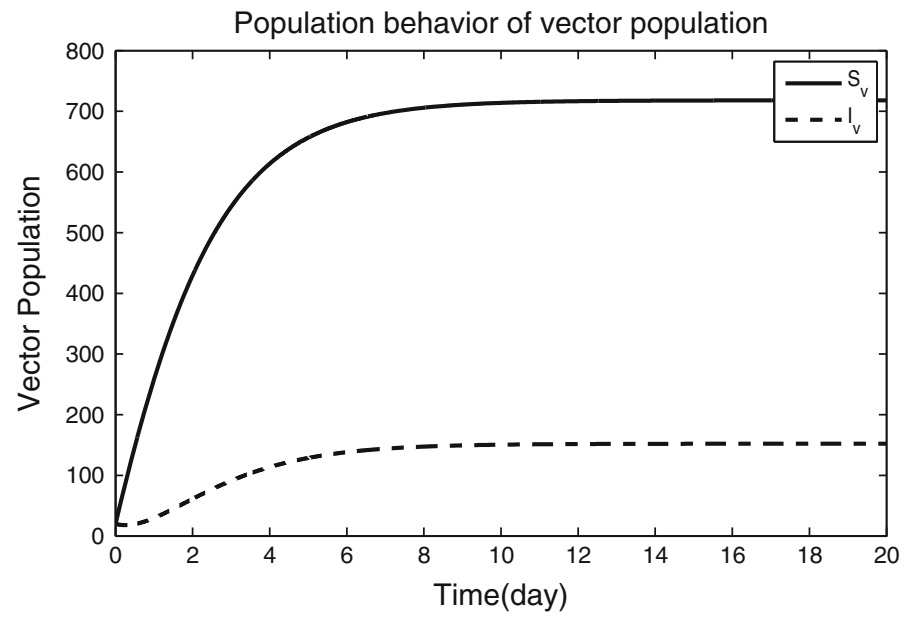

Fig. 3 Population behavior of vector

susceptible individuals change sharply. The plot shows the infected human population with the saturated effect of the values $\alpha_{1}$ and $\alpha_{2}$ in Fig. 5. The graph of infected human decreases with the saturated effect, for the values of $\alpha_{1}=0.83$ and $\alpha_{2}=0.83$. The dotted line shows the population of infected human without saturated effect and the dashed line shows the saturated effect. Due the effect of $\alpha_{1}$ and $\alpha_{2}$ the population of infected individuals changes. The plot shows the recovered human population with saturated effect for the values of $\alpha_{1}$ and $\alpha_{2}$ in Fig. 6. The graph of recovered human decreases with the saturated effect, for the values for $\alpha_{1}=0.83$ and $\alpha_{2}=0.83$. The dotted line shows the population of recovered human without saturated effect and the dashed line shows the saturated effect of human and the saturated effect of vector is represented by a dot dashed line. Due the effect of $\alpha_{1}$ and $\alpha_{2}$ the population of recovered individuals increases. The plot shows the vector population with saturated effect for the values of $\alpha_{1}$ and $\alpha_{2}$ in Fig. 7. The graph of susceptible vector 


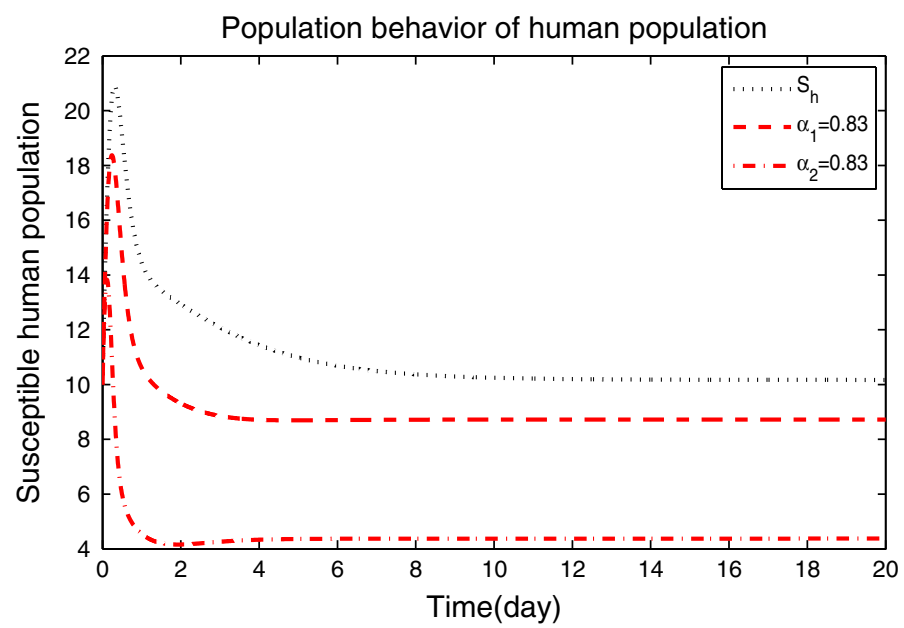

Fig. 4 Represents the graph of susceptible human with the effect of $\alpha_{1}$ and $\alpha_{2}$ with the parameters $\mu_{h}=0.31$, $b_{h}=099, \alpha 1=0.83, \alpha 2=0.083, \delta_{v}=0.32, \beta_{1}=0.91, b_{v}=290, \gamma_{h}=0.71, \gamma_{v}=0.8, \beta_{3}=0.22$, $\delta_{h}=0.61, \lambda_{h}=0.71, \beta_{2}=0.92$

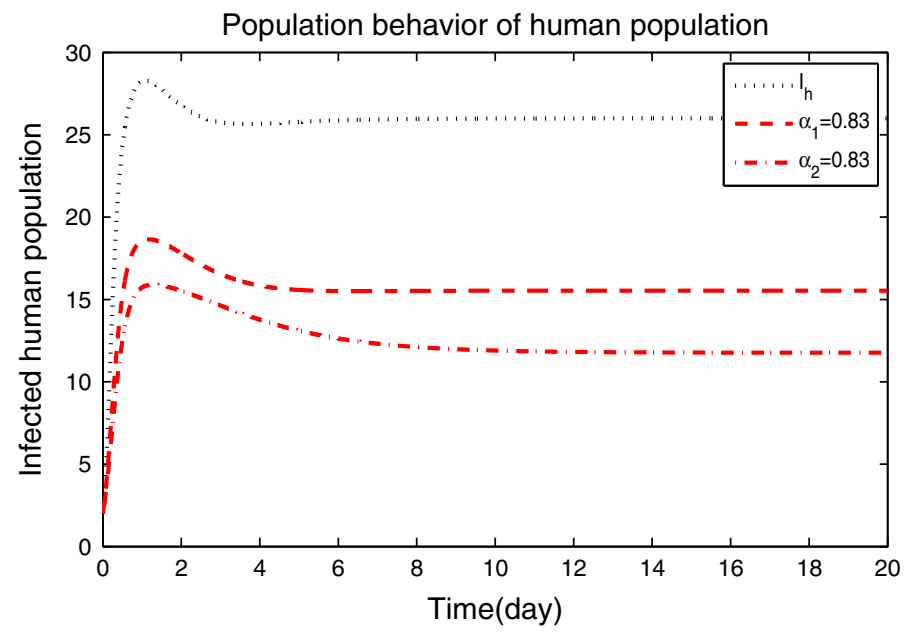

Fig. 5 Represents the graph of infected human with the effect of $\alpha_{1}$ and $\alpha_{2}$ with the parameters $\mu_{h}=0.31$, $b_{h}=099, \beta_{2}=0.92, \alpha 1=0.83, \alpha 2=0.083, \beta_{1}=0.91, \delta_{v}=0.32, b_{v}=290, \gamma_{h}=0.71, \gamma_{v}=0.8$, $\beta_{3}=0.22, \delta_{h}=0.61, \lambda_{h}=0.71$

decreases with the saturated infection rate, for the values for $\alpha_{1}=0.83$ and $\alpha_{2}=0.83$. The dotted line shows the population of susceptible human without saturated effect and the dashed line shows the saturated effect of human and the saturated effect of vector is represented by a dot dashed line. Due the effect of $\alpha_{1}$ and $\alpha_{2}$ the population of susceptible individuals decreases. The plot shows the infected vector population with the effect of $\alpha_{1}$ and $\alpha_{2}$ in Fig. 8. The graph of infected vector decreases with the with the saturation factor $\alpha_{1}=0.83$ and $\alpha_{2}=0.83$. The dotted line shows the population of infected vector without saturation effect and the dashed line shows the saturation effect of human and the saturated parameter effect of vector is represented by a dot dashed line. Due the effect of $\alpha_{1}$ and $\alpha_{2}$ the population 


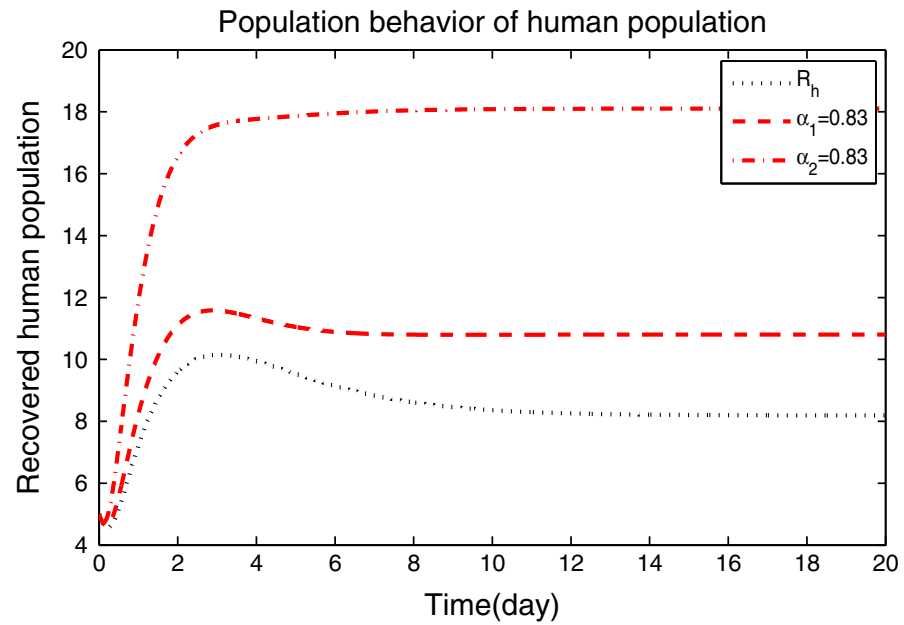

Fig. 6 Represents the graph of recovered human with the effect of $\alpha_{1}$ and $\alpha_{2}$ with the parameters $\mu_{h}=0.31$, $b_{h}=099, \beta_{1}=0.91, \alpha 1=0.83, \alpha 22=0.083, \delta_{v}=0.32, b_{v}=290, \gamma_{h}=0.71, \beta_{3}=0.22, \gamma_{v}=0.8$, $\beta_{2}=0.92, \delta_{h}=0.61, \lambda_{h}=0.71$

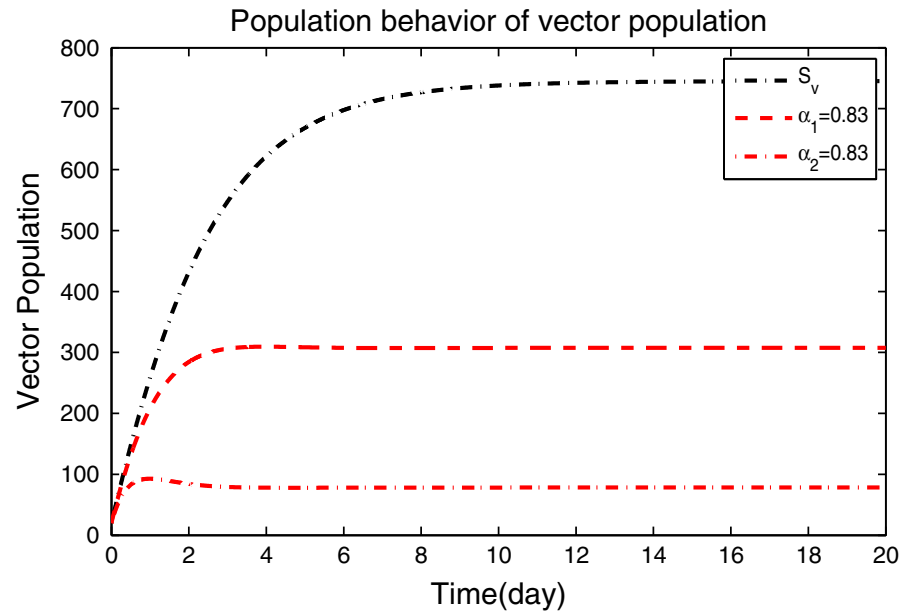

Fig. 7 Represents the graph of susceptible vector with the effect of $\alpha_{1}$ and $\alpha_{2}$ with the parameters $\mu_{h}=0.31$, $b_{h}=099, \alpha 1=0.83, \beta_{3}=0.22, \alpha 2=0.083, \delta_{v}=0.32, \beta_{1}=0.91, b_{v}=290, \gamma_{h}=0.71, \gamma_{v}=0.8$, $\beta_{2}=0.92, \delta_{h}=0.61, \lambda_{h}=0.71$

of infected vector decreases. The suggested parameters $\alpha_{1}=0.83$ and $\alpha_{2}=0.83$ can reduce the infection in the leptospirosis disease interaction with human.

\section{Conclusion}

The dynamic behavior of leptospirosis disease with saturated incidence has been presented successfully. The mathematical results obtained with their mathematical interpretation. The stability of the disease free and endemic equilibrium is completely described by the basic 


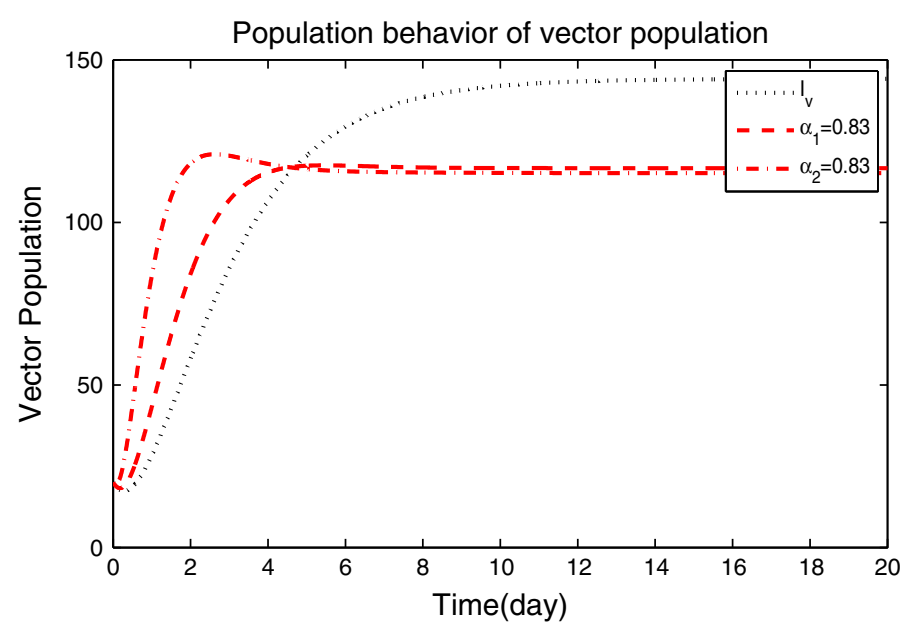

Fig. 8 Represents the graph of susceptible vector with the effect of $\alpha_{1}$ and $\alpha_{2}$ with the parameters $\mu_{h}=0.31$, $b_{h}=099, \alpha 1=0.83, \beta_{3}=0.22, \alpha 2=0.083, \delta_{v}=0.32, b_{v}=290, \gamma_{h}=0.71, \gamma_{v}=0.8, \beta_{1}=0.91$, $\delta_{h}=0.61, \lambda_{h}=0.71, \beta_{2}=0.92$

reproduction number $R_{0}$. We found, when $R_{0}<1$, the disease free equilibrium at $E_{0}$ is stable locally as well as globally. Further, the endemic equilibrium is obtained. We obtained that, when $R_{0}>1$, the endemic equilibrium is stable both locally and globally. Moreover, the numerical results for the model is obtained and briefly discussed. The parameters $\alpha_{1}$ and $\alpha_{2}$ effect have been discussed in numerical solution.

\section{References}

1. Abgueguen, P., Delbos, V., Blanvillain, J., Chennebault, J.M., Cottin, J., Fanello, S., et al.: Clinical aspects and prognostic factors of leptospirosis in adults. Retrospective study in France. J. Infect. 57, 171-178 (2008)

2. World Health Organization: Human Leptospirosis: Guidance for Diagnosis, Surveillance and Control. WHO, Geneva, pp. 1-109 (2003)

3. Adler, B., Faine, S.: The Genus Leptospira. The Prokaryotes. Springer, New York (2006)

4. Rafizah, Noor: A.A., et al.: Risk factors of leptospirosis among febrile hospital admissions in northeastern Malaysia. Prev. Med. 57, S11-S13 (2013)

5. Anderson, R.M., May, R.M.: Infectious Disease of Humans Dynamics and Control. Oxford University Press, Oxford (1991)

6. Ma, Z., Zhou, Y., Wu, J.: Modelling and Dynamics of Infectious Disease. Higher Education Press, Beijing (2009)

7. Okosun, K.O., Makinde, O.D.: A co-infection model of malaria and cholera diseases with optimal control. Math. Biosci. 258, 19-32 (2014)

8. Okosuna, K.O., Rachid, O., Marcus, N.: Optimal control strategies and cost-effectiveness analysis of a malaria model. BioSystems 111, 83-101 (2013)

9. Chitnis, N., Smith, T., Steketee, R.: A mathematical model for the dynamics of malaria in mosquitoes feeding on a heterogeneous host population. J. Biol. Dyn. 2, 259-285 (2008)

10. Derouich, M., Boutayeb, A.: Mathematical modelling and computer simulations of Dengue fever. App. Math. Comput. 177, 528-544 (2006)

11. Esteva, L., Vergas, C.: A model for dengue disease with variable human populations. J. Math. Biol. 38, 220-240 (1999)

12. Triampo, W., Baowan, D., Tang, I.M., Nuttavut, N., Wong-Ekkabut, J., Doungchawee, G.: A simple deterministic model for the spread of leptospirosis in Thailand. Int. J. Bio. Med. Sci. 2, 22-26 (2007) 
13. Zaman, G.: Dynamical behavior of leptospirosis disease and role of optimal control theory. Int. J. Math. Comput. 7(10), 80-92 (2010)

14. Pongsuumpun, P., Miami, T., Kongnuy, R.: Age structural transmission model for leptospirosis. In: The Third International Symposium on Biomedical Engineering, pp. 411-416 (2008)

15. Khan, M.A., Islam, S., Khan, S.A., Khan, I., Shafie, S., Gul, T.: Prevention of Leptospirosis infected vector and human population by multiple control variables. Abstr. Appl. Anal. 2014(3), 1-10 (2014)

16. Saddiq, S.F., Khan, M.A., Islam, S., Zaman, G., Jung, H., Khan, S.A.: Optimal control of an epidemic model of leptospirosis with nonlinear saturated incidences. Annu. Res. Rev. Biol. 4(3), 560-576 (2014)

17. Khan, M.A., Islam, S., Khan, S.A.: Mathematical modeling towards the dynamical interaction of leptospirosis. Appl. Math. Inf. Sci. 8(3), 1049-1056 (2014)

18. Ma, Z., Zhou, Y., Wang, W., Jin, Z.: Mathematicals Models and Dynamics of Infectious Diseases. China Science Press, Beijing (2004)

19. Xu, R., Ma, Z.: Global stability of a SIR epedemic model with time delay and nonlinear incidence. Nonlinear Anal. RWA 11, 3106-3109 (2010)

20. Capasso, V., Serio, G.: A generalization of the Kermack-McKendrick deterministic epidemic model. Math. Biosci. 42, 43-61 (1978)

21. Liu, W., Hethcote, H.W., Levin, S.A.: Dynamical behaviour of epidemiological models with non linear incidence rates. J. Math. Comput. 214(1), 2919-2926 (2009)

22. Saddiq, S.F., et al.: Optimal control of an epidemic model of leptospirosis with nonlinear saturated incidences. Annu. Res. Rev. Biol. 4(3), 560-576 (2014)

23. Leptospirosis information centre, www.leptospirosis.org

24. Lakshmikantham, V., leela, S., Martynyuk, A.A.: Stability Analysis of Non Linear Systems. Marcel. Dikker. Inc, New York (1989)

25. LaSalle, J.P.: The Stability of Dynamical Systems. SIAM, Philadelphia (1976)

26. Martin, R.: Logarithmic norms and projections applied to linear differential systems. J. Math. Anal. Appl. 45, 432-454 (1974)

27. Li, M.Y., Muldowney, J.S.: A geometric approach to global-stability problems. SIAM J. Math. Anal. 27, 1070-1083 (1996)

28. Tangkanakul, W., Smits, H.L., Jatanasen, S., Ashford, D.A.: An emerging health problem in Thailand, South Asian. J. Trop. Med. Pub. Health 36, 281-288 (2005) 